\title{
Painful supernumerary phantom arm following motor cortex stimulation for central poststroke pain
}

\section{Case report}

\section{Sergio Canavero, M.D., Vincenzo Bonicalzi, M.D., Giancarlo Castellano, M.D., Paula Perozzo, Psychol.D., and Barbara Massa-Micon, M.D. \\ Pain Relief Unit, Department of Neurosciences, Service of Nuclear Medicine, and Neuropsychology Unit, University of Turin, Ospedale Molinette, Turin, Italy}

In this report, the authors describe a case in which the patient began to experience a supernumerary phantom arm after she received motor cortex stimulation for central pain. The patient had a history of right thalamocapsular stroke. It is speculated that the motor cortex activation triggered a response in the patient's parietal lobe, precipitating perception of the phantom limb.

To the authors' knowledge this is the first reported case of its kind.

Key Words * cortical stimulation * central pain * phantom limb

Supernumarary phantom limbs (pseudopolymyelia) are seldom reported.[1,7-10,13,14,16-23] They indicate a neurological symptom of stroke and generally occur on the right side. They may also be present with head or spinal cord injury, brain tumor, and multiple sclerosis.

We describe a woman in whom central pain developed poststroke. Following motor cortex stimulation (MCS) performed to ease the pain, the patient began to experience a supernumerary arm hallucination. No similar case appears in the literature.

\section{CASE REPORT}

History. This 61-year-old woman had previously experienced hypertensive hemorrhage in the right side of her brain, which involved the thalamus, internal capsule, and deep subparietal corona radiata, and coursed into the ventricles (Fig. 1). 

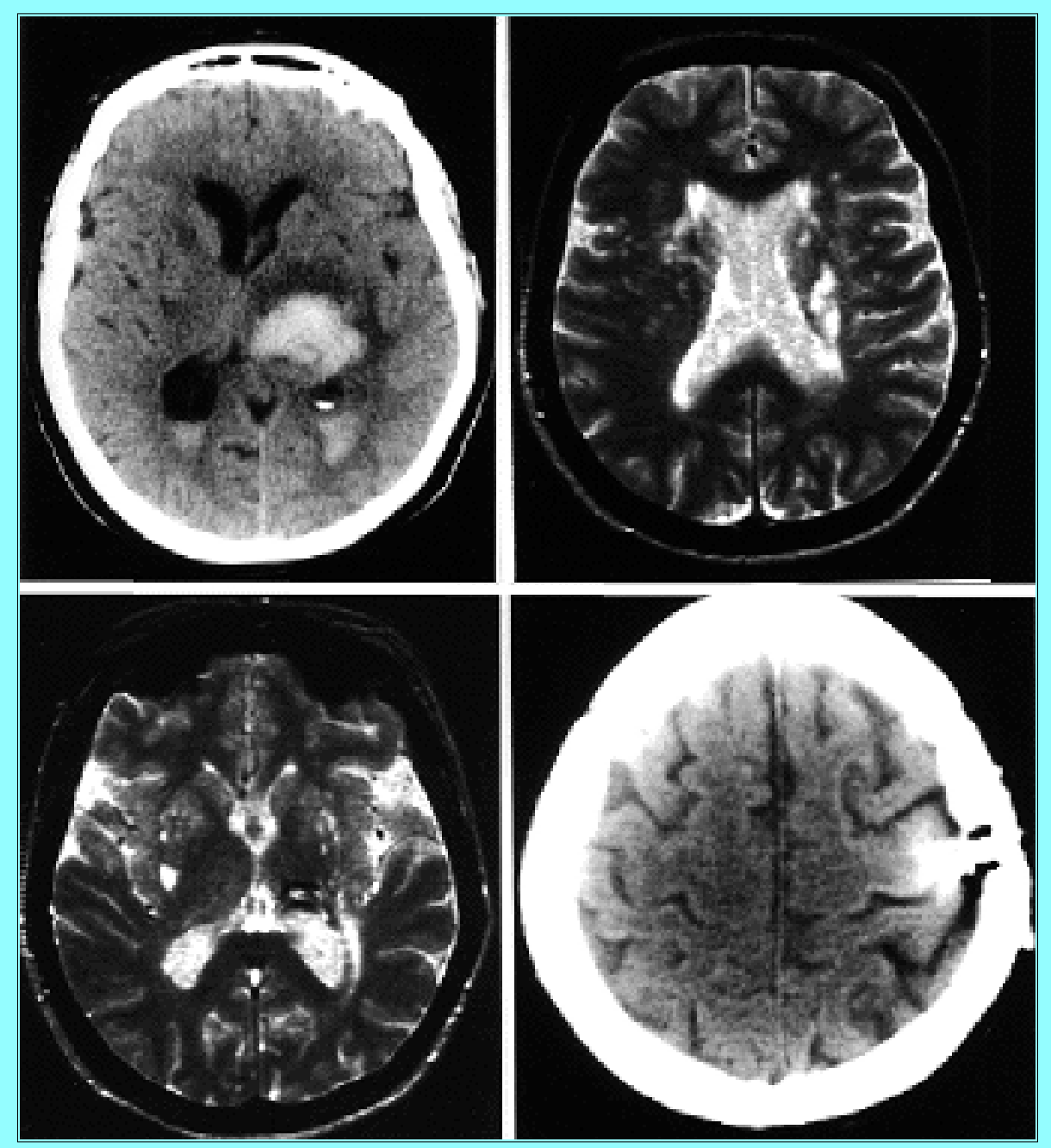

Fig. 1. Computerized tomography (upper left) and magnetic resonance (upper right and lower left) images demonstrating evidence of the patient's hemorrhagic stroke. Another computerized tomography scan revealing the motor cortex stimulator in place (lower right).

Examination. On admission 3 years later (1998), the patient's neurological examination disclosed a plegic left arm, a paretic left leg that hampered her gait, and tactile-pinprick anesthesia of the left side. The patient complained of a ripping, squeezing, and burning pain in the left half of her body, mostly occurring in the face, arm, and leg; allodynia was also present.

Treatment. The woman's pain was completely relieved when a subhypnotic propofol test was administered, but not when placebo was substituted (Class A/gamma-aminobutyric acid-responsive central pain[4]). Her pain also was relieved when, according to our protocol,[4] extradural cortical stimulation to the primary motor cortex was applied.[3] The ripping and squeezing pains were totally relieved and the burning pain was diminished by half. Unfortunately, the burning sensation could not be controlled further during a 5-week treatment period, despite the fact that parameter adjustments were made to the stimulator.

Phantom Limb. Approximately 6 weeks after implantation of the stimulator (May 1998), the patient spontaneously volunteered that she experienced the presence of a painful supernumerary left arm, which she unhesitantly called "my phantom arm." This phantom limb was sensed once every 1 to 2 weeks. The experience lasted 10 to 15 minutes and sometimes longer; resolution of the pain was always abrupt and spontaneous. The stimulator was switched off because the patient obtained no more relief and the pain was worsened by changing the stimulator parameters into the high-voltage range. The patient's central 
pain syndrome relapsed. Some relief was later obtained using a course of high-dose mexiletine and gabapentin.

As part of our neurostimulation protocol, both neurometabolic and neuropsychological assessments (State Trait Anger Expression Inventory and Cognitive Behavioral Assessment [version 2]) were made. High-resolution single-photon emission computerized tomography, performed using hexamethylpropylenamine oxide and Iomazeil, revealed right thalamic and temporal hypoperfusion, which was normalized by the MCS. On examination, the woman appeared fully oriented, coherent, alert, and cooperative. She had no history of epilepsy or psychiatric disorders, and she had never experienced hallucinations, including phantom sensations, prior to stimulation. Her cognition appeared normal. The autotopagnosia was normal, and there was no anosognosia of her impaired limbs. In addition there was no anosodiaphoria, disorder of calculation, interpretative elaboration, delusion, or reduplication of people, places, or events. Instead, there was reactive depression, introversion, difficulty with social interaction, confabulation, right-left confusion, and high levels of inhibited aggression. She would shy away from people, being hypersensitive to criticism and external judgment. However, questions concerning her phantom limb did not cause her discomfort.

The phantom limb was described as originating from the shoulder. This phantom arm reportedly was flexed and externally rotated; it never moved spontaneously. The sensation of the phantom limb was always accompanied by pain having a crushing quality. When present, it made the patient's left arm pain worse. Touch or other stimulation could not evoke the phantom sensations. Instead, when the left arm was passively moved, the phantom limb moved also. Interestingly, on these occasions, the patient's left arm pain was made more tolerable. During the follow-up period (6 months), the patient could also feel her phantom arm as it seemed to stretch straight forward in front of her.

\section{DISCUSSION}

Supernumerary phantom appendages can be understood in the general context of a central brain mechanism.[2] Ad hoc hypotheses range from psychological (resolution of cognitive dissonance, symbolic elaboration, delusional misidentification) to organic (sensory deprivation and perceptual neuronal completion). However, data do show that phantom pain is not a function of emotional adjustment.[12]

All cases of supernumerary phantom limb hallucinations are accompanied, to a variable degree, by both sensory and motor impairment. Although the importance of denervation is well recognized as far as phantom percepts are involved, this phenomenon alone cannot explain pseudopolymyelia.[22] A recent study found that resolution of this reduplication syndrome was paired with an improvement in a patient's right-left laterality discrimination and other tasks of mental orientation in space, whereas the patient's sensory and motor functions did not significantly improve.[22] The fact that supernumerary phantom limbs are experienced more frequently after right-sided brain stroke[14] is closely akin to the higher proportion of central pain after right thalamic stroke[4]--the right hemisphere appears to have a role in monitoring somatic states and a dominant role in processing somatic representation (see references cited in recent correspondence[5]). Attentional networks may play an additional role.[9]

Halligan and colleagues[13] maintain that the emergence of supernumerary phantom limbs is determined more by motor loss than by sensory loss. The role of the motor system in this context is supported by the present case, in which MCS appears to have triggered the phantom sensations. We observed no effect of MCS on the patient's plegic arm, but her left foot seemed to "keep ahead of" her. In a functional 
magnetic resonance imaging study, Ersland and associates[11] reported activation of the primary motor cortex by phantom limb fingertapping. Kew and colleagues [15] found positron emission tomography evidence of an increased metabolism in both primary motor and sensory cortices in patients with postamputation phantom percepts, but not in congenitally limbless patients with no complaints of phantom sensations.[15] Those patients who can control the movements of their phantom limbs can sometimes reduce the associated pain.[25]

We speculate that MCS set off the oscillatory corticothalamic mechanism responsible for our patient's subjective awareness of her arm (see Discussion in another study[2]). This speculation hinges on the parietal lobe, including that portion responsible for sensorimotor integration and maintenance of the internal representation of the body state.[24] To generate stable percepts, the brain weighs evidence from many different sources. If a conflict is generated, a loop is activated and a perception of a supernumerary limb ensues. In this schema, illusory movements are explained by the cooperative action of both sensory and motor cortical areas. In theory, incorporation of this schema into the idea of Crick and Koch[6] that awareness is subtended by a link between primary sensory areas and motor areas may be possible.

\section{CONCLUSIONS}

Supernumerary phantom limbs highlight brain mechanisms of consciousness. Their occurrence in the context of cortical stimulation for central pain supports the notion that these anomalous phenomena are the result of an unbalanced information flow between the thalamus and cortex (dynamic reverberation[2]).

\section{References}

1. Bechterev VM: [Partial cortical and subcortical paralyses of psychoreflexive functions.] Obozr Psykhiatr:31-41, 1926 [uncorrected draft]

2. Canavero S: Dynamic reverberation. A unified mechanism for central and phantom pain. Med Hypotheses 42:203-207, 1994

3. Canavero S, Bonicalzi V: Cortical stimulation for central pain. J Neurosurg 83:1117, 1995 (Letter)

4. Canavero S, Bonicalzi V: The neurochemistry of central pain: evidence from clinical studies, hypothesis and therapeutic implications. Pain 74:109-114, 1998

5. Canavero S, Bonicalzi V: Pain after thalamic stroke: right diencephalic predominance and clinical features in 180 patients. Neurology 51:927-928, 1998

6. Crick F, Koch C: Are we aware of neural activity in primary visual cortex? Nature 375:121-123, 1995

7. Critchley M: The Parietal Lobes. New York: Hafner, 1966

8. Davis R: Pain and suffering following spinal cord injury. Clin Orthop 112:76-80, 1975

9. Donnet A, Schmitt A, Poncet M, et al: [Hallucinations in supernumerary limbs, left hemineglect and hypersexuality in a case of right capsulo-lenticular hematoma.] Rev Neurol 153:587-590, 1997 (Fr)

10. Ehrenwald H: Verändertes Erleben des Körperbildes mit konsekutiver Wahnbildung bei linsksseitiger Hemiplegie. Monatschr Psychiatr Neurol 75:89-97, 1930 
11. Ersland L, Rosen G, Lundervold A, et al: Phantom limb imaginary fingertapping causes primary motor cortex activation: an fMRI study. Neuroreport 8:207-210, 1996

12. Fisher K, Hanspal RS: Phantom pain, anxiety, depression, and their relation in consecutive patients with amputated limbs: case reports. Br Med J 316:903-904, 1998

13. Halligan PW, Marshall JC: Supernumerary phantom limb after right hemispheric stroke. J Neurol Neurosurg Psychiatry 59:341-342, 1995

14. Halligan PW, Marshall JC, Wade DT: Three arms: a case study of supernumerary phantom limb after right hemisphere stroke. J Neurol Neurosurg Psychiatry 56:159-166, 1993

15. Kew JJM, Ridding MC, Rothwell JC, et al: Reorganization of cortical blood flow and transcranial magnetic stimulation maps in human subjects after upper limb amputation. J Neurophysiol 72:2517-2524, 1994

16. Mayeux R, Benson DF: Phantom limb and multiple sclerosis. Neurology 29:724-726, 1979

17. Ohry A, Gur S, Zeilig G: 'Duplicate limbs' sensation in acute traumatic quadriplegia. Paraplegia 27:257-260, 1989

18. Pineas H: Ein Fall von phantomähnlichen Erscheinungen ("Phantomarm") bei hemiplegischer Lähmung. Nervenartzt 5:233-236, 1932

19. Rogers MJC, Franzen MD: Delusional reduplication following closed-head injury. Brain Inj 6:469-476, 1992

20. Sellal F, Renaseau-Leclerc C, Labrecque R: L'homme à six bras. Un examen de membres fantômes surnuméraires après ramollissement sylvien droit. Rev Neurol (Paris) 152:190-195, 1996

21. Van Bogaert L: Sur la pathologie de l'image de soi. (Etudes anatomodiniques.) Ann Medicopsychol 92:519-744, 1934

22. Vuilleumier P, Reverdin A, Landis T: Four legs. Illusory reduplication of the lower limbs after bilateral parietal lobe damage. Arch Neurol 54:1543-1547, 1997

23. Weinstein EA, Kahn RL, Malitz S, et al: Delusional reduplication of parts of the body. Brain 77:45-60, 1954

24. Wolpert DM, Goodbody SJ, Husain M: Maintaining internal representations: the role of the human superior parietal lobe. Nat Neurosci 1:529-533, 1998

25. Wynn Parry CB: Pain in avulsion lesions of the brachial plexus. Pain 9:41-53, 1980

Manuscript received November 20, 1998.

Accepted in final form February 22, 1999.

Address reprint requests to: Sergio Canavero, M.D., Via Montemagno 46, 10132 Turin, Italy. 